\title{
Breast cancers with high DSS1 expression that potentially maintains BRCA2 stability have poor prognosis in the relapse-free survival
}

Andri Rezano ${ }^{1}$, Kazuhiko Kuwahara ${ }^{1}$, Mutsuko Yamamoto-lbusuki ${ }^{2}$, Masahiro Kitabatake ${ }^{1}$, Penpak Moolthiya ${ }^{1}$, Suchada Phimsen', Taiji Suda ${ }^{3}$, Shigenobu Tone ${ }^{3}$, Yutaka Yamamoto², Hirotaka Iwase ${ }^{2}$ and Nobuo Sakaguchi ${ }^{*}$

\begin{abstract}
Background: Genetic BRCA2 insufficiency is associated with breast cancer development; however, in sporadic breast cancer cases, high BRCA2 expression is paradoxically correlated with poor prognosis. Because DSS1, a mammalian component of the transcription/RNA export complex, is known to stabilize BRCA2, we investigated how the expression of DSS1 is associated with clinical parameters in breast cancers.

Methods: DSS1 mRNA and p53 protein were examined by RT-PCR and immunohistochemical staining of breast cancer specimens to classify DSS1 $7^{\text {high }}$ and DSS1 $1^{\text {low }}$ or p53 high and $\mathrm{p} 53^{\text {low }}$ groups. Patient survival was compared using Kaplan-Meier method. DSS $1^{\text {high }}$ or DSS $7^{\text {low }}$ breast cancer cells were prepared by retroviral cDNA transfection or DSS1 siRNA on proliferation, cell cycle progression, and survival by flow cytometric analyses with or without anti-cancer drugs.

Results: In comparison to patients with low levels of DSS1, high-DSS1 patients showed a poorer prognosis, with respect to relapse-free survival period. The effect of DSS1 was examined in breast cancer cells in vitro. DSS1 high-expression reduces the susceptibility of MCF7 cells to DNA-damaging drugs, as observed in cell cycle and apoptosis analyses. DSS1 knockdown, however, increased the susceptibility to the DNA-damaging drugs camptothecin and etoposide and caused early apoptosis in p53 wild type MCF7 and p53-insufficient MDA-MB-231 cells. DSS1 knockdown suppresses the proliferation of drug-resistant MDA-MB-231 breast cancer cells, particularly effectively in combination with DNA-damaging agents.

Conclusion: Breast cancers with high DSS1 expression have worse prognosis and shorter relapse-free survival times. DSS1 is necessary to rescue cells from DNA damage, but high DSS1 expression increases drug resistance. We suggest that DSS1 expression could be a useful marker for drug resistance in breast cancers, and DSS1 knockdown can induce tumor apoptosis when used in combination with DNA-damaging drugs.
\end{abstract}

Keywords: Breast cancer, BRCA2, Cancer prognosis, DNA damage, DSS1

\section{Background}

Breast cancer development is associated with various molecular abnormalities in genes involved in DNA repair, cell cycle control, signal transduction, and tumor suppressor function; these are the predisposing hereditary causes in approximately $5-10 \%$ of breast cancers [1]. Hereditary breast cancers exhibit germline mutations of BRCA1 and

\footnotetext{
* Correspondence: nobusaka@kumamoto-u.ac.jp

${ }^{1}$ Department of Immunology, Graduate School of Medical Sciences,

Kumamoto University, 1-1-1, Honjo, Chuo-ku, Kumamoto 860-8556, Japan Full list of author information is available at the end of the article
}

BRCA2 at certain incidences. Most breast cancer cases with germline BRCA2 mutations have loss of heterozygosity at the $B R C A 2$ locus, resulting in the loss of the $B R C A 2$ allele [2,3]. BRCA2 deficiency is associated with various abnormalities in the response to DNA cross-linking agents, such as defects in homologous recombination (HR), formation of RAD51 foci, DNA replication, and checkpoint regulation [4-9].

In contrast, in the majority (90\%) of sporadic breast cancers, BRCA2 is not mutated [10]. Rather, the expression of BRCA2 is increased in tumors, as shown in reverse

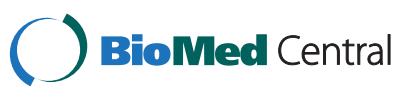


transcription (RT)-PCR, quantitative RT-PCR (qRT-PCR), and immunohistochemical analyses [11]. BRCA2 is significantly over-expressed in sporadic breast, ovarian, pancreatic, and prostatic cancers [12]. BRCA2 over-expression, but not decreased expression, was correlated with histopathological grade III; this over-expression, which is attributable to nuclear polymorphism, was also correlated with the mitotic index, implicating a close association between BRCA2 over-expression and the proliferation rate of breast cancer cells [11,13]. Furthermore, a three-gene expression signature (BRCA2, DNMTB3, and CCNEI) was found to be an independent prognostic marker in breast cancer [14]. A high BRCA2 level is associated with poor outcome and correlated with high proliferation rate. In a hierarchical clustering analysis of 47 candidate genes, BRCA2 was found to be the leading gene in a cluster of proliferation-associated genes. The finding was supported by an in vitro study in which BRCA2 over-expression suppressed HR and reduced RAD51 foci formation, along with inactivation of $\mathrm{p} 53$, which suggests that moderate levels of BRCA2 play a role in the stimulation of HR for appropriate DNA repair [15].

The expression level of BRCA2 is presumably regulated through various mechanisms including transcription, subcellular localization, binding to partners, and protein modification and stabilization. A stabilization factor of BRCA2, deleted in split hand/split foot 1 (DSS1), was originally identified as a BRCA2-associated protein, and its depletion was shown to induce BRCA2 destabilization [16]. DSS1 is a candidate gene for an inherited limb development disease and is located on chromosome 7q21.3q22.1. DSS1 is a principal component of the mammalian mRNA transcription/exportation 2 (TREX2) complex that includes GANP, PCID2, and DSS1 and interacts with various components of RNA metabolism including RNA polymerase II, RNA splicing factors, and helicases [17]. Saccharomyces deficient in the components of the TREX2 complex displayed abnormalities in cell proliferation and cell cycle control, but abnormal expression of individual components of TREX2 results in different phenotypes in mammalian cells. For example, mammalian GANP insufficiency causes DNA injuries during proliferation and is associated with tumor development in human glioblastoma [18]. Loss of PCID, another TREX2 component, causes a severe defect in Mad2 expression with a marked reduction in Mad2 mRNA export, which causes severe hyperploidy and apoptotic cell death [19]. However, increased expression of TREX2 components, in contrast to reduced expression, has rarely been shown to be associated with tumor development.

Given that the BRCA2-expression is correlated with poor prognosis in clinical cases [11,13], we investigated the outcome of abnormal DSS1 expression in human breast cancer cases. DSS1 is certainly expressed at high levels in a group of breast cancer cases with poor prognosis. The imbalance of DSS1 over-expression associated with BRCA2 expression could affect breast cancer development. Here, we demonstrate that increased DSS1 expression is correlated with chemo-resistance in sporadic breast cancers, which might be responsible for the worse prognosis of patients with high DSS1 levels, particularly with respect to relapse-free survival (RFS).

\section{Methods}

\section{Patients and breast cancer tissues}

Breast tumor specimens from 289 female patients with invasive breast carcinoma, who were treated at Kumamoto University Hospital between 2001 and 2009, were included in this study. Among these patients, p53 immunohistochemical data were available for 227 (78.5\%) patients. The patients were from a consecutive series; those with other malignancies or bilateral breast cancer were excluded. Samples were snap frozen in liquid nitrogen at the time of the pretherapeutic biopsy or surgical treatment and stored at $-80^{\circ} \mathrm{C}$ until simultaneous total RNA extraction. The median age of the patients was 59 years (range, 21-93 years). Adjuvant treatment and neoadjuvant treatment were decided by risk evaluation according to tumor biology [estrogen receptor alpha $(E R \alpha)$, progesterone receptor (PgR), and HER2 but not Ki-67 status] and clinical staging, including sentinel lymph node biopsy, in accordance with the recommendations of the St. Gallen international expert consensus on the primary therapy of early breast cancer. In detail, neoadjuvant treatments were administered to 62 patients, 46 of whom received chemotherapy and 16 of whom received hormonal therapy. The breast-conserving rate was $68.2 \%$, and most of these were treated with radiotherapy. Axillary lymph node dissection was carried out in $45.2 \%$ of cases; the others were spared dissection due to negative lymph node status by sentinel node exploration. A total of 208 patients were treated with hormone therapy, 106 patients were given chemotherapy, and 19 patients were treated with trastuzumab. The ethics committee of Graduate School of Medical Sciences, Kumamoto University approved the study protocol. Informed consent was obtained from all patients. Patients received follow-up studies every 3 months. The median follow-up period was 66 months (range 15-144 months).

\section{RNA extraction, primers, and qRT-PCR}

Total RNA was isolated from tissue specimens using the Allprep DNA/RNA Mini Kit (Qiagen, Germantown, $\mathrm{MD})$. Total RNA $(0.5 \mu \mathrm{g})$ was reverse transcribed to cDNA using PrimeScript RT reagent Kit (Takara Bio Inc., Otsu, Japan), according to the manufacturer's protocol. Each PCR was performed using $2 \mu$ of cDNA and $0.2 \mu \mathrm{mol} / \mathrm{l}$ of each probe in a LightCycler System with SYBR Premix Dimer Eraser (Takara Bio Inc.). PCR primer 
sequences were as follows: for DSS1, forward 5'-GTTA GAGGAAGACGACGAGT-3' and reverse 5'-GGATGCT ATGAAGTCTCCAT-3'; for $\beta$-actin, forward $5^{\prime}$-TGGCA CCCAGCACAATGAA-3' and reverse 5' -CTAAGTCA TAGTCCGCCTAGAAGCA-3'. Each reaction $(20 \mu \mathrm{l}$ samples) was performed under the following conditions: initialization for $10 \mathrm{sec}$ at $95^{\circ} \mathrm{C}$, and then 45 cycles of amplification, with $5 \mathrm{sec}$ at $95^{\circ} \mathrm{C}$ for denaturation and $20 \mathrm{sec}$ at $60^{\circ} \mathrm{C}$ for annealing and elongation. The expression level of DSS1 mRNA is given as relative copy numbers normalized to those of $\beta$-actin mRNA. In some experiments, qRT-PCR was performed using a LightCycler (Roche Diagnostics, Indianapolis, IN). Specific oligonucleotide primers and probes for DSS1 and gapdh were purchased (Nihon Gene Research Laboratories, Sendai, Japan). The specific primers for DSS1 were the same as above. Their sequences were as follows: dss 1 donor probe, 5' -CCCAATTATCCTCCCAGACATGTGCATC TT-3'; dss1 acceptor probe, 5'-ATCTTCATCTAAGC CAGCCCAGTCTTCGG-3'; gapdh-F, 5-CAGCCTCAA GATCATCAGC-3'; gapdh-R, 5'-GGCCATCCACAGT CTTCT-3'; gapdh donor probe, 5'-GGTCATCCAT GACAACTTTGGTATCGTGGAA-3'; gapdh acceptor probe, 5'-GACTCATGACCACAGTCCATGCCATCA CTG-3'. The level of DSS1 mRNA expression was determined relative to gapdh.

\section{Immunohistochemistry and scoring system}

Histological sections $(4 \mu \mathrm{m})$ were deparaffinized and incubated for $10 \mathrm{~min}$ in methanol containing $0.3 \%$ hydrogen peroxide. Mouse monoclonal antibodies (mAbs) against ER $\alpha$ (SP1, Ventana Japan, Tokyo, Japan), PgR (1E2, Ventana Japan) and Ki67 (MIB1, Dako Japan, Tokyo, Japan), a polyclonal Ab against HER2 (Dako Japan), and a mouse mAb against p53 (DO7, Dako Japan) were used; staining was carried out in the NexES IHC Immunostainer (Ventana Medical Systems, Tucson, AZ), in accordance with the manufacturer's instructions. ER $\alpha$ and PgR status were evaluated based on the percentage of positively stained nuclei, and the status of a nucleus was considered positive when $\geq 1 \%$ of the nucleus was stained. HER2 was evaluated using the HercepTest method (Dako Japan), with membranous staining scored on a scale of 0 to $3+$. Tumors with scores of $\geq 3$ or with a $\geq 2.2$-fold increase in HER2 gene amplification as determined by fluorescence in situ hybridization were considered to be positive for HER2 over-expression. Ki67 was scored as the percentage of nuclear-stained cells out of all cancer cells along the invasive front of the tumor in $\times 400$ high-power fields; this gave the Ki67 labeling index. p53 was evaluated based on the percentage of positively stained nuclei, and the status of a nucleus was considered positive when $\geq 20 \%$ of the nucleus was stained.

\section{Cell lines and small interfering RNA (siRNA) treatment}

The human breast cancer cell lines MCF7 and MDAMB-231 were obtained from the American Type Culture Collection and maintained in RPMI-1640 (Life Technologies, Carlsbad, CA) supplemented with 10\% heatinactivated fetal calf serum (MP Biomedicals, Santa Ana, CA), $2 \mathrm{mM}$ L-glutamine (Lonza, Allendale, NJ), and $5 \times$ $10^{-5} \mathrm{M}$ 2-mercaptoethanol (Wako Pure Chemicals Industries, Osaka, Japan) with $5 \% \mathrm{CO}_{2}$ at $37^{\circ} \mathrm{C}$. siRNA treatment was performed when the cells reached 50\% confluence; cells were transfected with $10 \mathrm{nM}$ of control (siCtrl) or DSS1 ( $\mathrm{siDSS1}$; Life Technologies) siRNA using Lipofectamine RNAiMAX (Life Technologies). The siRNA target sequences were as follows: siDSS1-(a), 5'-GACAAUGUAGAGGAUGACU UCUCUA-3'; siDSS1-(b), 5'-GCAGCCGGUAGACUU AGGUAUGUUA-3'; siCtrl-(a), 5'-GACGUAUAGGGU AAGUCCUUAACUA-3'; siCtrl-(b), 5' -GCAGGCGA UUCAGAUCUGGUGCUUA-3'. The results with siDS S1-(a) are shown in the figures as representatives.

\section{Establishment of DSS1 over-expressing cells}

A retroviral vector, designated pFB-DSS1-IRES-GFP, was transfected into PLAT-GP (Cellbiolabs, San Diego, CA) retrovirus packaging cells using FuGENE HD (Roche Diagnostics). MCF7 and MDA-MB-231 cells were infected with the retroviruses using polybrene $(8 \mu \mathrm{g} / \mathrm{ml}$; Sigma, St. Louis, MO) for 2 days. GFP-positive cells were sorted using a JSAN cell sorter (BayBioscience, Kobe, Japan).

\section{Cell proliferation assay}

In vitro cell proliferation was determined using an MTT assay performed with a Cell Proliferation Kit I (Roche Applied Science, Penzberg, Germany). Briefly, at $24 \mathrm{hr}$ after siRNA treatment, $2.5 \times 10^{3}$ cells per well were seeded and incubated with MTT labeling reagent $(0.5 \mathrm{mg} / \mathrm{ml})$ for $6 \mathrm{hr}$ at $37^{\circ} \mathrm{C}$. The soluble formazan product was quantified using an ELISA reader at $590 \mathrm{~nm}$ from day 1 to day 4 .

\section{Detection of dead cells}

DNA damage-inducing agents were added when the cells reached $80 \%$ confluence. CPT (Merck Millipore, Billerica, MA) or ETP (Merck Millipore) was introduced to the cells at a final concentration of $50 \mu \mathrm{M}$. After 24 to $72 \mathrm{hr}$, cells were harvested, washed with ice-cold PBS, resuspended in PBS with $250 \mu \mathrm{g} / \mathrm{ml}$ RNase A, and stained with $2 \times$ PI solution for $2 \mathrm{hr}$ at $4^{\circ} \mathrm{C}$. The cell cycle was analyzed using FACSCalibur (BD, Franklin Lakes, NJ) and CellQuest software.

\section{Annexin XII/PI staining}

Cells were washed twice with staining buffer (0.1 M Hepes $\mathrm{pH} 7.4,1.4 \mathrm{M} \mathrm{NaCl}, 25 \mathrm{mM} \mathrm{CaCl}_{2}$ ) and resuspended in 
FITC-conjugated Annexin XII (Abcam, Cambridge, UK) on ice for $10 \mathrm{~min}$. Cells were then counterstained with $0.5 \mu \mathrm{g} / \mathrm{ml}$ PI on ice for $5 \mathrm{~min}$, and an equal volume of staining buffer was added. Early apoptotic (Annexin XII ${ }^{+} / \mathrm{PI}^{-}$) and late apoptotic/necrotic (Annexin $\mathrm{XII}^{+} / \mathrm{PI}^{+}$) cells are shown with merged color.

\section{EM analysis}

After DSS1 knockdown, floating cells were centrifuged in microfuge tubes and then fixed in $2.5 \%$ glutaraldehyde in $0.1 \mathrm{M}$ cacodylate buffer $(\mathrm{pH} 7.2)$ containing $0.1 \%$ $\mathrm{CaCl}_{2}$. After washing in the same buffer, embedding in $2 \%$ agarose and incubating post-fixation in $1 \% \mathrm{OsO}_{4}$ for $1 \mathrm{hr}$, the specimens were washed again, dehydrated in a series of ethanol baths, and embedded in Epon 812. Thin sections were cut with a Leica Ultracut UCT microtome, post-stained with $2 \%$ uranyl acetate and Reynolds lead citrate, and examined using a JEOL JEM 1400 operated at $80 \mathrm{kV}$. Two researchers (TS and ST) independently analyzed cells with apoptotic changes using digitized EM images.

\section{Alkaline comet assay}

DNA damage induced by DSBs and single-strand breaks were analyzed using a comet assay kit (Trevigen, Inc., Gaithersburg, MD), according to the manufacturer's protocol. For quantification, the tail moment (an index of DNA damage calculated as the product of the tail length and the fraction of DNA in the comet tail) was evaluated using CometScore version 1.5 software (AutoComet.com). At least a hundred cells from each sample were scored.

\section{Statistical analysis}

The nonparametric Wilcoxon (for uni-variable), KruskalWallis test (for multi-variables), and the $x^{2}$ test were adopted for statistical analysis of the associations between the DSS1 mRNA status and various clinicopathological factors. RFS and BCSS curves were calculated according to the Kaplan-Meier method and verified by the log-rank test. All statistical significance was defined as $P<0.05$. JMP software version 8.0.2 for Windows (SAS institute Japan, Tokyo, Japan) was used for all statistical analyses.

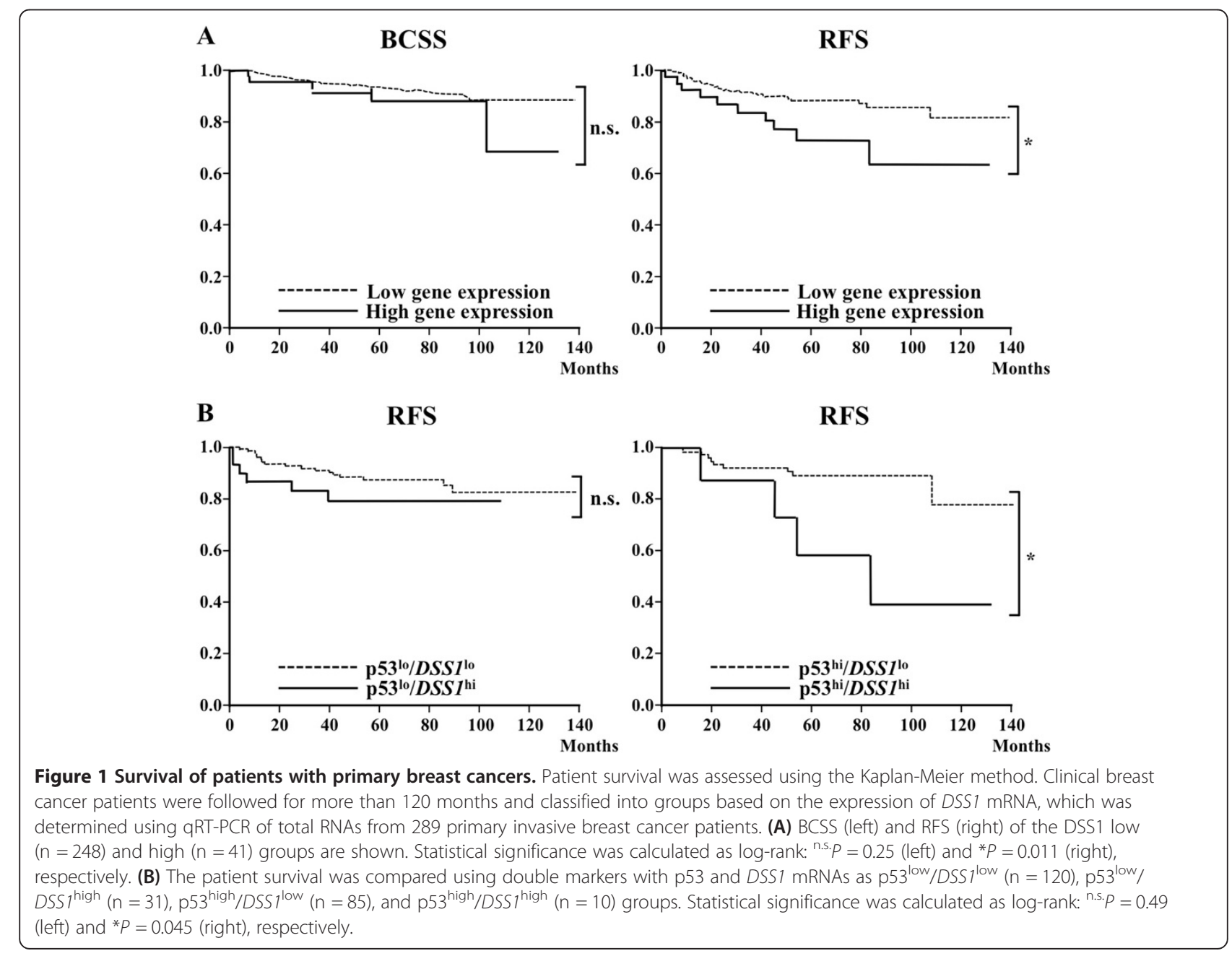




\section{Results}

Increased DSS1 expression in breast cancer cases with poor prognosis

We examined the survival of breast cancer patients using the Kaplan-Meier method. We analyzed the expression level of DSS1 mRNA by qRT-PCR from the tumor samples obtained at surgery using primers for DSS1 mRNA (Additional file 1: Figure S1). The relative expression level was arbitrarily determined and used for classification of breast cancer cases into DSS1-high ( $\left.D S S 1^{\text {high }}\right)$ and DSS1-low $\left(D S S 1^{\text {low }}\right)$ groups. The $D S S 1^{\text {high }}$ group showed a significant shorter survival time ( $P=0.011)$ on the RFS curve, while the difference in the breast cancer specific survival (BCSS) curves was not significant (n.s.; $P=0.25$ ) (Figure 1A). DSS1 physically interacts with the RPN3/S3 proteasomal subunit, which increases the degradation of ubiquitinated p53 [20]. In a large cohort, over-expression of p53 was significantly associated with poor prognosis in premenopausal women treated with tamoxifen after chemotherapy [21]. The p53 expression was used for classification by arbitrarily determined conditions (see Methods). We compared RFS among p $53^{\text {low } / ~}$ $D S S 1^{\text {low }}, \mathrm{p} 53^{\text {high }} / D S S 1^{\text {low }}, \mathrm{p} 53^{\text {low }} / D S S 1^{\text {high }}$, and $\mathrm{p} 53^{\text {high }} /$ $D S S 1^{\text {high }}$ groups (Figure $1 \mathrm{~B}$ ). While the number of cases was small, no change of the survival curve was observed when p53 expression was low; however, the DSS1 $1^{\text {high }}$ group showed a worse prognosis in comparison with the $D S S 1^{\text {low }}$ group in breast cancer cases with high $\mathrm{p} 53$ expression $\left({ }^{*} P=0.045\right)$. Other parameters and markers were compared in the $D S S 1^{\text {high }}$ and $D S S 1^{\text {low }}$ groups (Table 1). No significant differences were observed in the groups of pre- or post-menopausal women, positive or negative nodal status, tumor size $<20$ or $\geq 20 \mathrm{~mm}$, nuclear grade I to III, positive or negative vessel invasion score, $\mathrm{ER} \alpha$ status, and PgR status. However, DSS1 expression showed a significant difference $\left({ }^{*} P=0.0255\right)$ based on HER2 status, which is presumably associated with effective treatment using trastuzumab as the standard regimen for HER2-positive breast cancer cases. Interestingly, DSS1 expression was markedly increased (55.6; median of DSS1 relative expression) in the group of breast cancer cases with low Ki67 labeling indices $(<20 \%)$ compared with the expression (36.0; median of DSS1 relative expression) in the group with high Ki67 labeling indices ( $\geq 20 \%)$.

The data suggest that the high DSS1 expression may not be directly associated with the proliferation of breast cancer cells in humans.

\section{Effect of increased DSS1 expression on resistance to chemotherapy}

For molecular analysis, we used two breast cancer cell lines, MCF7 (ER $\alpha^{+} \mathrm{PgR}^{+}$with wild type p53) and MDAMB-231 (ER $\alpha^{-}$PgR $^{-}$with p53 mutation). The effect of DSS1 over-expression on cell proliferation and cell cycle progression was examined in breast cancer cells that had been engineered to over-express DSS1 $\left(\mathrm{MCF}^{\mathrm{DSS} 1}\right.$ or

Table 1 Comparison of DSS1 gene expression among various groups of breast cancer patients

\begin{tabular}{|c|c|c|c|c|}
\hline Clinical parameters & Group & Number of patient & DSS1 gene expression median $(25 \%, 75 \%)$ & Mann-Whitney U-test $(P)$ \\
\hline \multirow[t]{2}{*}{ Menopause } & Pre- & 78 & $48.7(8.05,143)$ & \multirow[t]{2}{*}{0.554} \\
\hline & Post- & 210 & $49.2(8.09,92.4)$ & \\
\hline Nodal & $(-)$ & 179 & $41.2(7.04,94.2)$ & \multirow[t]{2}{*}{0.132} \\
\hline status & $(+)$ & 110 & $64.9(13.2,117)$ & \\
\hline Tumor & $<20$ & 125 & $44.3(8.08,108)$ & \multirow[t]{2}{*}{0.646} \\
\hline size $(\mathrm{mm})$ & $\geq 20$ & 164 & $52.2(8.41,104)$ & \\
\hline Nuclear & 1 & 143 & $51.7(8.33,114)$ & \multirow[t]{3}{*}{0.703} \\
\hline \multirow[t]{2}{*}{ grade } & 2 & 74 & $39.7(8.24,90.0)$ & \\
\hline & 3 & 72 & $57.5(6.19,91.3)$ & \\
\hline Vessel & $(-)$ & 187 & $40.0(5.47,88.9)$ & 0.308 \\
\hline invasion & $(+)$ & 77 & $47.1(11.5,107)$ & \\
\hline \multirow[t]{2}{*}{ ERa } & $(-)$ & 65 & $52.7(5.71,105)$ & \multirow[t]{2}{*}{0.962} \\
\hline & $(+)$ & 224 & $49.0(8.36,102)$ & \\
\hline \multirow[t]{2}{*}{$\mathrm{PgR}$} & $(-)$ & 96 & $60.3(6.14,103)$ & \multirow[t]{2}{*}{0.487} \\
\hline & $(+)$ & 193 & $47.1(8.37,106)$ & \\
\hline \multirow[t]{2}{*}{ HER2 } & $(-)$ & 248 & $53.2(10.6,115)$ & \multirow[t]{2}{*}{${ }^{*} 0.0255$} \\
\hline & $(+)$ & 41 & $21.8(3.78,74.8)$ & \\
\hline Ki67 & $<20$ & 113 & $55.6(16.4,132)$ & \multirow[t]{2}{*}{$* * 0.0096$} \\
\hline (\%) & $\geq 20$ & 169 & $36.0(4.97,82.0)$ & \\
\hline
\end{tabular}

$(*)$ and $\left({ }^{* *}\right)$ denote $P<0.05$ and $P<0.01$, respectively. 
MDA-MB-231 ${ }^{\mathrm{DSS} 1}$ ) by retroviral transfection (Additional file 2: Figure S2). Stable transfectants that expressed high levels of DSS1 mRNA did not show any changes in cell cycle or cell proliferation compared with the control (GFP only) transfectants (Figure 2A). DSS1-over-expressing cells showed no abnormalities in proliferation. Trastuzumab is a first choice for treatment of HER2-positive breast cancer in our cohort, but camptothecin (CPT) is often chosen as a regular regimen for HER2-negative breast cancer cases (Table 1). The susceptibility of DSS1-overexpressing breast cancer cells to the DNA-damage inducing agents CPT and etoposide (ETP) was examined. DSS1 over-expression renders breast cancer cells more resistant to treatment with $\mathrm{CPT}$, an inhibitor of type 1 topoisomerase (which cleaves one strand of doublestranded DNA), compared with the GFP-control MCF7 transfectants (Figure 2B), while no change was detected in DSS1 over-expressing MDA-MB-231 transfectants (Figure 2C). The effect of DSS1 over-expression was masked in both MCF7 and MDA-MB-231 cells upon exposure to ETP, an inhibitor of topoisomerase II (which cleaves two strands of double-stranded DNA and induces cell cycle arrest at G2/M) (Additional file 3: Figure S3A and $\mathrm{B})$. Therefore, DNA damage was examined using the alkaline comet assay (Figure 3A and B). DSS1 overexpression reduced CPT-induced DNA damage in MCF7

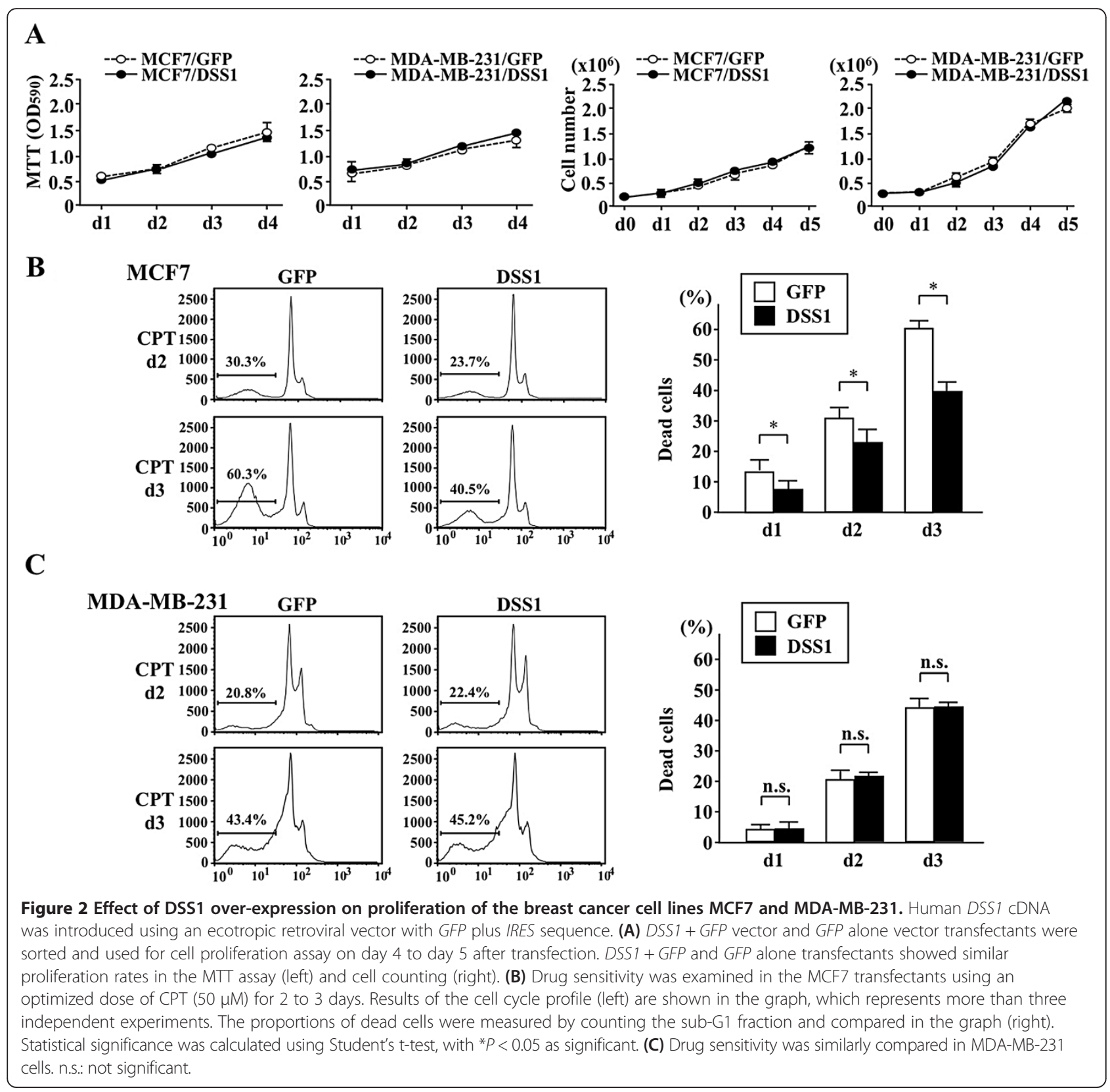




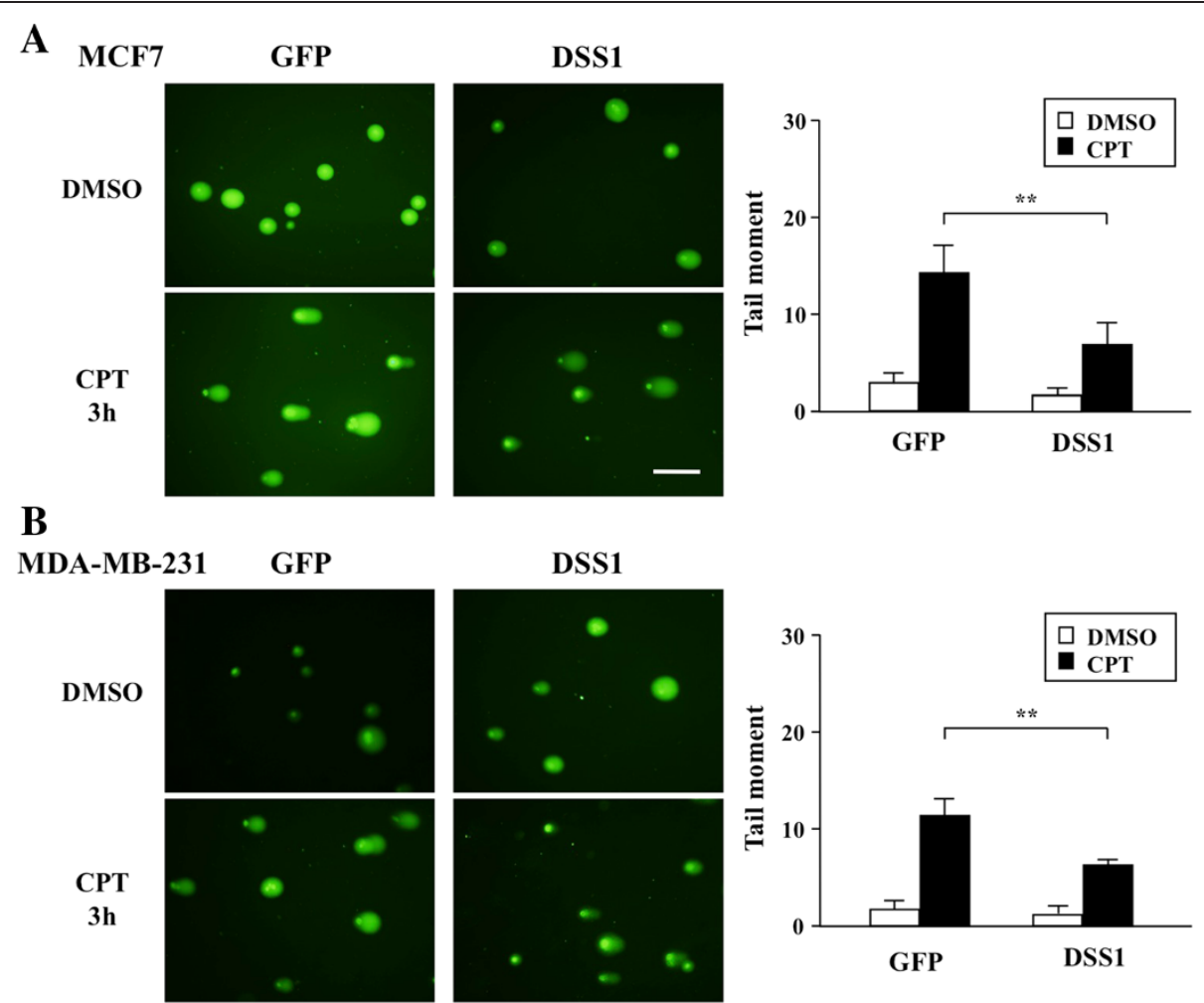

Figure 3 Effect of DSS1 over-expression on sensitivity to DNA damage. Effect of DSS1 over-expression on drug-induced DNA injury was measured using the alkaline comet assay after treatment of cells with CPT (50 $\mu \mathrm{M})$ for $3 \mathrm{hr}$. DMSO was used as a solvent control. The DNA content pattern observed under fluorescent microscopy is shown (left), and the DNA damage rate was measured as the tail moment in the fluorescence image using CometScore software. DSS-over-expressing MCF7 (A) or MDA-MB-231 (B) cells were compared with GFP-expressing controls. Data obtained by counting more than 100 cells are shown as a graph, which represents more than three independent experiments (right). Statistical significance was calculated using Student's t-test, with ${ }^{* *} P<0.01$ as significant. Scale bar indicates $100 \mu \mathrm{m}$.

and MDA-MB-231 cells, suggesting that DSS1 overexpression increases drug resistance in breast cancers.

Effect of DSS1 knockdown on the sensitivity of breast cancer cells carrying wild-type p53 or insufficient p53 to chemotherapeutics

Next, we examined cell growth in DSS1-depleted cells. Two siRNAs directed against two independent DSS1 mRNA sequences elicited marked reductions in DSS1 mRNA levels (Additional file 4: Figure S4A) and protein levels (Additional files 4: Figure S4B and 5); we therefore used one of these sequences in the following experiments. DSS1 knockdown (siDSS1) caused decreased cell growth, as observed in an MTT assay (Figure 4A: left) and in an assessment of cell number (right). At 3 and 5 days after siDSS1-treatment, the number of dead cells in the sub-G1 population increased compared to siCtrltreated cells (Figure 4B). siDSS1-treated MCF7 cells experienced cell cycle arrest at $M$ phase entry, with increases in $\mathrm{G} 2 / \mathrm{M}$ phase populations at day 5 (27.0\% vs. $6.20 \%)$. siDSS1 also markedly induced the number of sub-G1 (dead) cells in drug-resistant MDA-MB-231 (24.8\%, compared with $3.99 \%$ for siCtrl).
Treatment of MCF7 cells with siDSS1 caused apoptosis, as detected by staining with Annexin XII and propidium iodide (PI). Representative Annexin $\mathrm{XII}^{+} \mathrm{PI}^{-}$ (early apoptotic) and Annexin $\mathrm{XII}^{+} \mathrm{PI}^{+}$(late apoptotic/ necrotic) cells are shown in Figure 5A. We further examined cell morphology at day 5 after siDSS1 treatment using electron microscopy (EM). siDSS1 treatment induced cell death in MCF7 cells, along with typical apoptotic changes including chromatin condensation, enlarged nuclei and smaller nuclei compared to the siCtrl-treated cells (Figure 5B; chromatin condensation marked with arrowheads). siDSS1 treatment also caused apoptotic cell death in MDA-MB-231, with condensed chromatin and smaller nuclei relative to the control cells (Figure 5B; marked with arrowheads); however, some of these cells also showed atypical cell death morphologies, with enlarged cell size accompanied by electron microscopically void nuclei containing finely condensed chromatin-like structures adjacent to the inner nuclear membrane (Figure 5B; marked with asterisks). Because MDA-MB-231 do not seem to arrest at M phase entry, presumably due to p53 mutation, the atypical cell death observed in siDSS1-treated MDA-MB-231 might represent 


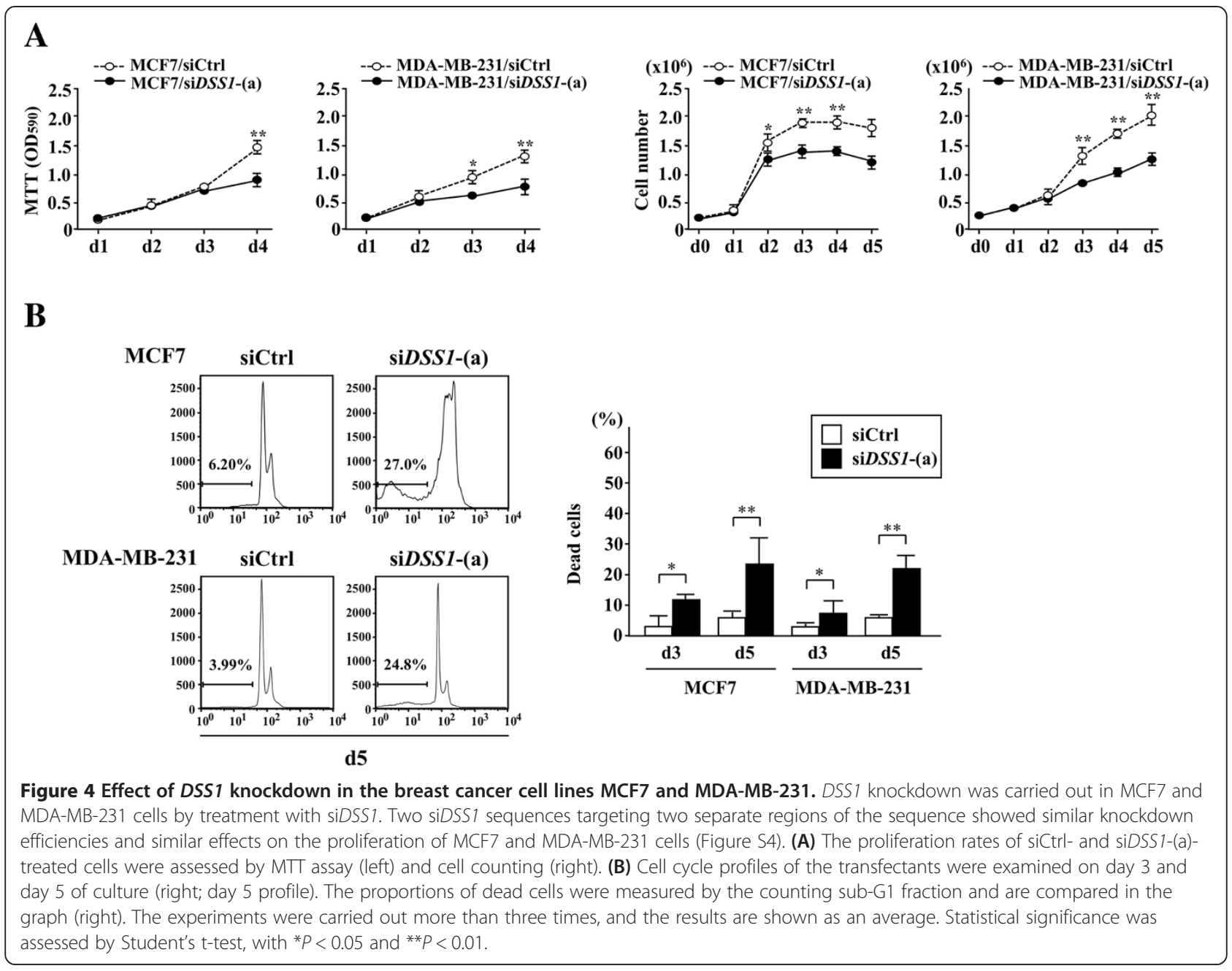

cell death occurring at various cell cycle stages. Finally, while the cell morphologies were not identical, siDSS1treated MCF7 and MDA-MB-231 cells showed similar extents of DNA damage in the alkaline comet assay (Figure 5C).

To confirm the effect of DSS1 on breast cancer cells, we investigated the effect of DSS1 knockdown on cell cycle and cell death in breast cancer cells during early stages of treatment with CPT or ETP. DSS1 knockdown markedly increased the number of cells in the sub-G1 apoptotic population in both MCF7 and MDA-MB-231 cells treated with CPT as early as day 2 (Figure 6A). Similarly, DSS1 knockdown increased the sub-G1 population in both breast cancer cell lines treated with ETP (Figure 6B). Because MDA-MB-231 cells, which are of mesenchymal origin and have a triple-negative phenotype [22], are well known to be resistant to several DNA-damaging agents, these data suggested that DSS1 depletion can increase chemosensitivity in drugresistant breast cancer cells with either wild type or mutant p53.

\section{Discussion}

Here, we demonstrate for the first time that DSS1 overexpression can be used as an early diagnostic marker for poor prognosis in cases of breast cancer. Because DSS1 maintains BRCA2 expression by regulating its ubiquitindependent degradation [23] and because the BRCA1: BRCA2 imbalance promotes tumorigenesis by increasing genomic instability [24], we predicted that increased expression of DSS1 could suppress breast cancer development. However, unexpected results have been observed in breast cancer cases. BRCA2 over-expression, rather than decreased expression, is associated with a poor survival rate [13] and poorer histological findings [11] in breast cancer cohorts. Our finding agrees with previous observations in cervical cancers with high DSS1 expression [25] and in breast cancers with high BRCA2 expression $[11,13,14]$. In a cervical cancer cohort [25], human papilloma virus oncoprotein E6 was found to bind to p53 and targets it for degradation through the ubiquitin-proteasome system, which also regulates BRCA2 stabilization. The expression of DSS1 is upregulated in 


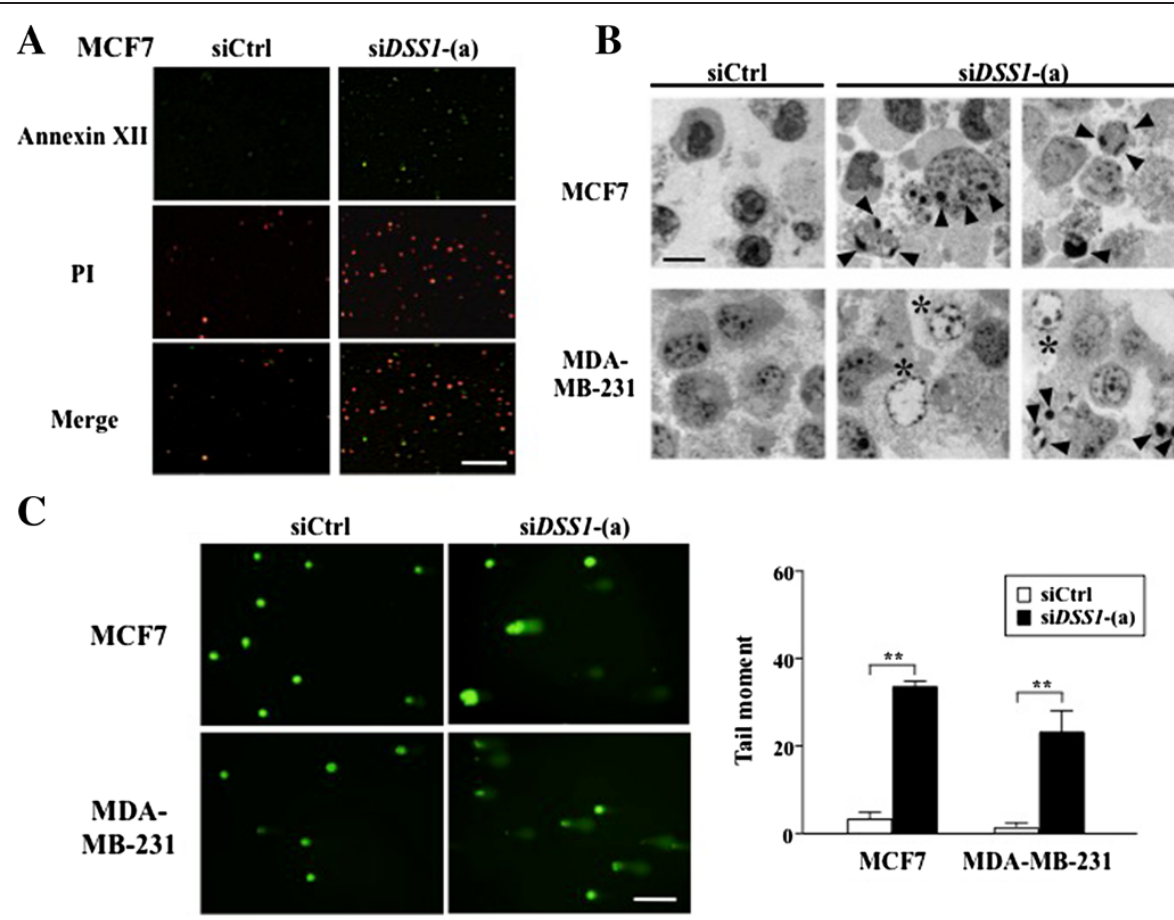

Figure 5 Effect of DSS1 knockdown on apoptotic cell death in breast cancer cells. (A) To examine the stage of cell death, live cells were stained with Annexin XII, which identifies the early apoptotic stage, in combination with PI staining; cells were marked as Annexin $\mathrm{XII}^{+} \mathrm{PI}{ }^{-}$ (green; early apoptotic), Annexin $\mathrm{XI}^{+} \mathrm{PI}^{+}$(doubly merged yellow; the intermediate stage), or Annexin $\mathrm{XII}^{-} \mathrm{PI}^{+}$(red; late apoptotic/necrotic). Scale bar indicates $100 \mu \mathrm{m}$. (B) EM analysis was carried out at day 5 after treatment with siCtrl or siDSS1-(a). Arrowheads show the chromatin condensation typical of cell death, and asterisks indicate cell death with atypical chromatin accumulation and density. Scale bar indicates $500 \mu \mathrm{m}$. (C) An alkaline comet assay was carried out to detect the DNA damage resulting from both single-strand and double-strand breaks and evaluated as in Figure 3. Statistical significance was assessed by Student's t-test, with ${ }^{*} P<0.01$. Scale bar indicates $200 \mu \mathrm{m}$.

cancerous regions compared to normal ones. The high DSS1 expression that potentially maintains high-level BRCA2 expression might cause or enhance breast cancer proliferation and/or drug resistance.

Cells with insufficient levels of $B R C A$ genes are unable to repair DNA damage during the cell cycle and will eventually die. Tumors with germline BRCA mutations accompany the capacity to escape cell cycle checkpoints, which is in accordance with the low incidence of mutant BRCA-mediated sporadic tumorigenesis. BRCA2 interacts with RAD51 and BRCA1 to mediate DNA repair, $\mathrm{P} / \mathrm{CAF}$ to mediate histone acetylation, BRAF-35 to mediate cell cycle regulation, and DSS1 [26]. DNA double-stranded breaks (DSBs) cause severe DNA damage, leading to replication arrest. BRCA1 and BRCA2 are involved in the repair of DSBs through their interaction with RAD51. BRCA2 is primarily involved in HR repair, but BRCA1 is involved in alternative DNA repair by nonhomologous end joining (NHEJ) [27]. These DNA repair pathways are important for maintaining the integrity of the genome against the DSBs that occur at various phases of the cell cycle [28]. HR is principally involved in an errorfree DNA repair mechanism that protects the genome during the $\mathrm{S}$ phase of cell cycle when the allelic genes on chromosomes are in close proximity [29]. Transcriptioncoupled DNA damage occurring at the other phases of the cell cycle is repaired by the NHEJ, which is required for prompt DNA repair in rapidly proliferating cells regardless of small errors in the genome [30]. The cellular genome is presumably maintained by the balanced regulation of these two DNA repair mechanisms [31].

The human DSS1 is homologous to Sem1, a component of the Saccharomyces TREX2 complex, which is composed of Sac3/Thp1/Sem1 in Saccharomyces cerevisiae. Thus, it is likely to be a functional component of the GANP/ PCID2/ENY2/centrin/DSS1 complex involved in mRNA export in mammalian cells [32]. Many studies have addressed the character and function of the individual components of TREX2 complex in mammals [33]. Loss of any component of the mammalian TREX2 complex elicited a defect either in mRNA export or in the regulation of cell cycle progression, implying that each TREX2 component has an important individual function. Most of the defects associated with loss of the TREX2 component involve either replication or survival. In particular, DSS1 plays a role in stabilization of BRCA2 through regulation of its 


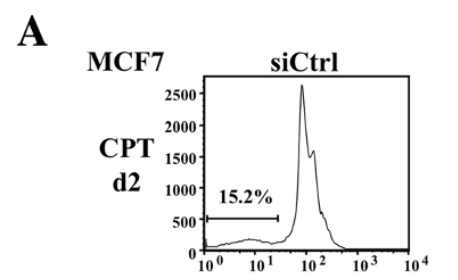

MDA-MB-231

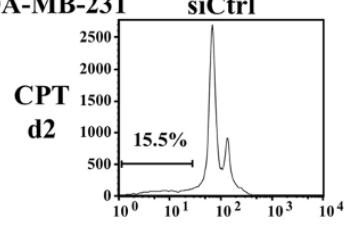

B

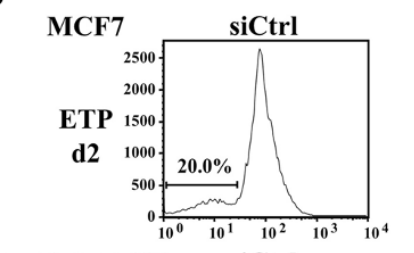

MDA-MB-231

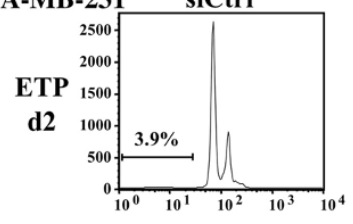

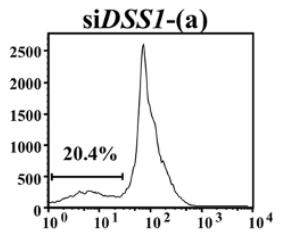

siDSS1-(a)
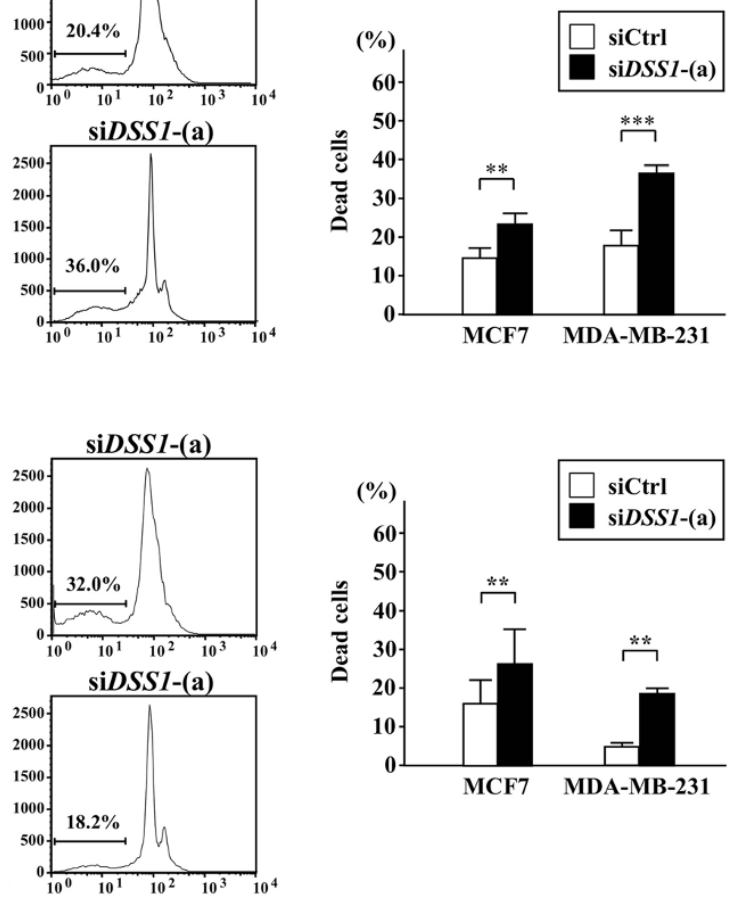

Figure 6 Effect of DSS1 knockdown on the drug sensitivity of MCF7 and MDA-MB-231 cells. Drug sensitivity was examined in DSS1 knockdown cells with an optimized dose of CPT $(50 \mu \mathrm{M})$ and ETP $(50 \mu \mathrm{M})$. MCF7 and MDA-MB-231 cells were first treated with siDSS1 for 2 days and then cultured with CPT (A) or ETP (B) for 2 days. (A) and (B) The number of cells in the sub-G1 (dead) cell populations was compared between siDSS1-(a)- or siCtrl-treated cells. The results of cell cycle profiling (left) were converted into the graph, which represents more than three independent experiments (right). Statistical significance was assessed by Student's t-test, with ${ }^{* *} P<0.01$ and ${ }^{* * *} P<0.001$.

ubiquitin-dependent proteolytic degradation [23]. This may suggest a unique function for DSS1 in the organization of the ribonucleoprotein complex during various processes: transcription, nuclear to cytoplasmic export, and translation.

The $\mathrm{p} 53^{\text {high }} / D S S 1^{\text {high }}$ group showed a worse prognosis in comparison with the $\mathrm{p} 53^{\text {low }} / D S S 1^{\text {high }}$ group in breast cancer cases (Figure 1B), suggesting that the increased p53 status accelerates the effect of DSS1 over-expression on tumor progression under regular clinical treatments. This effect was not simply represented in the CPTtreatment of tumor cells between p53-wild type MCF7 ${ }^{\mathrm{DSS} 1}$ and p53-mutated MDA-MB-231 ${ }^{\mathrm{DSS} 1}$ (Figure 2C), while both of which sustained similar DNA damages by CPT under DSS1 over-expression (Figure 3). siDSS1, however, rendered both tumor cells very sensitive to CPT and ETP (Figure 6), suggesting that the effect of siDSS1 appears dominantly on chemosensitivity of breast cancer cells irrespective with TP53 status in the short-term culture. DSS1 might be involved not only in the proteolytic degradation of p53 [20], the stabilization of BRCA2 [23] and the process of DNA repair but also in the cell survival against anti-cancer drugs.

Regarding the cause of the poor prognosis in high DSS1-expressing breast cancers, an intriguing observation is the marked enhancement of drug sensitivity in highly drug-resistant MDA-MB-231 breast cancer cells by DSS1 knockdown (Figure 4). Drug resistance is caused by various biological mechanisms in cancer cells, including pharmacokinetic resistance and/or cellular resistance. Beside the cellular drug transporter systems that allow multidrug resistance of breast cancer cells, decreases in various nuclear enzymes could also cause multi-drug resistance. Decreased activity of topoisomerases has been described in several drug-resistant cancer cells including breast cancers [34]. Defects in cellular signaling pathways leading to apoptosis and DNA repair could result in multi-drug resistance, as can p53 insufficiency, decreases in ceramide levels [35], DNA alkyl-transferase activation [36], and problems with the mismatch DNA repair system $[37,38]$. Decreased expression of BRCA2 has been suggested to be a marker for a favorable response to docetaxel in breast 
cancer [39]. BRCA2 knockdown has been proposed to as a means to synergistically potentiate cisplatin and melphalan treatment [40]. High DSS1 expression, which potentially stabilizes BRCA2 and maintains cancer cell proliferation, could increase drug resistance under the standard clinical regimens of breast cancer treatment, presumably resulting in decreased disease-free survival.

\section{Conclusions}

Sporadic cases of breast cancer with high expression of DSS1 showed a worse prognosis with respect to RFS; however, increased DSS1 expression was not correlated with increased proliferation or tumor grade. DSS1 over-expression increased the resistance of breast cancer cells to DNA-damaging drugs; conversely, DSS1 knockdown rendered breast cancer cells more sensitive to these drugs. These results clearly indicate that DSS1 knockdown in combination with chemotherapy might be effective for treatment of breast cancer.

\section{Additional files}

Additional file 1: Figure S1. Differences between the DSS1 $1^{\text {high }}$ and the DSS1 ${ }^{\text {low }}$ groups based on qRT-PCR. Patients having tumors with high DSS1 expression were classified by the mRNA level as the DSS $1^{\text {high }}$ group (DSS1/ $\beta$-actin ratio $>136$ ). Boxes represent the mean and $70 \%$ confidence intervals; bars, standard deviations.

Additional file 2: Figure S2. Establishment of DSS1 over-expressed MCF7 and MDA-MB-231 cells. (A) Schematic diagram of retroviral vectors (pFB-IRES-GFP and pFB-DSS1-IRES-GFP). (B) Increased expression of DSS1 transcripts in DSS1 over-expressed MCF7 and MDA-MB-231 cells. Representative data is shown from three independent experiments.

Additional file 3: Figure S3. Drug sensitivity in DSS1 over-expressed MCF7 and MDA-MB-231 cells. (A) and (B) The effect of DSS1 overexpression on drug sensitivity was examined in MCF7 and MDA-MB-231 cells at day 2 and day 3 after treatment with ETP $(50 \mu \mathrm{M})$. n.s.: not significant.

Additional file 4: Figure S4. Effect of siDSS1 on DSS1 expression and cell proliferation. (A) siDSS1s on two independent sequences were transfected into MCF7 and MDA-MB-231 cells. The expression levels of DSS1 transcripts were measured by GRT-PCR. Similar knockdown efficiency was observed in both siDSS1-(a) and siDSS1-(b) transfected cells. The data are representative of three independent experiments. (B) The expression levels of DSS1 were measured by Western blot in siCtrl-, siDSS1-(a)-, and siDSS1-(b)-treated cells. $\beta$-actin was used as a loading control. (C) Effect of siDSS1-(b) was similar in cell proliferation (MTT assay) compared with that of siDSS1-(a) shown in Figure 4. Statistical significance is shown by the Student's t-test calculation with ${ }^{* *} P<0.01$ and ${ }^{* *} P<0.001$

Additional file 5: Western blot analysis.

\section{Abbreviations}

HR: Homologous recombination; RT-PCR: Reverse transcription-PCR; qRTPCR: Quantitative RT-PCR; DSS1: Deleted in split hand/split foot 1; TREX2: Transcription/exportation 2; RFS: Relapse-free survival; BCSS: Breast cancer specific survival; ERa: Estrogen receptor a; PgR: Progesterone receptor; CPT: Camptothecin; ETP: Etoposide; NHEJ: Non-homologous end joining; siRNA: Small interfering RNA.

\section{Competing interests}

The authors declare that they have no competing interests.

\section{Authors' contributions}

AR, KK, MY-I, MK, PM, SP, TS, and ST acquired data and performed the statistical analysis. MY-I, YY, and HI performed the cohort analysis. KK designed the study, analyzed and interpreted the data, and drafted the manuscript. NS designed the study, analyzed and interpreted the data, and drafted and approved the manuscript. All authors read and approved the final manuscript.

\section{Acknowledgments}

We would like to thank Mika Ito for technical assistance. This work was supported by Grant-in Aid from the Ministry of Education, Culture, Sports, Science, and Technology in Japan (KK and NS) and by a contract research fund from the Program of Founding Research Centers for Emerging and Reemerging Infectious Diseases (NS). AR is supported by an Indonesian Directorate General of Higher Education (DIKTI) Scholarship.

\section{Author details}

${ }^{1}$ Department of Immunology, Graduate School of Medical Sciences, Kumamoto University, 1-1-1, Honjo, Chuo-ku, Kumamoto 860-8556, Japan. ${ }^{2}$ Department of Breast and Endocrine Surgery, Graduate School of Medical Sciences, Kumamoto University, 1-1-1, Honjo, Chuo-ku, Kumamoto 860-8556, Japan. ${ }^{3}$ Department of Biochemistry and Electron Microscope Center, Kawasaki Medical School, 577 Matsushima, Kurashiki, Okayama 701-0192, Japan.

Received: 26 June 2013 Accepted: 26 November 2013 Published: 1 December 2013

\section{References}

1. Balmain A, Gray J, Ponder B: The genetics and genomics of cancer. Nat Genet 2003, 33:238-244.

2. Collins N, McManus R, Wooster R, Mangion J, Seal S, Lakhani SR, Ormiston W, Daly PA, Ford D, Easton DF, et al: Consistent loss of the wild type allele in breast cancers from a family linked to the BRCA2 gene on chromosome 13q12-13. Oncogene 1995, 10:1673-1675.

3. Gudmundsson J, Johnnesdottir G, Bergthorsson JT, Arason A, Ingvarsson S, Egilsson V, Barkardottir RB: Different tumor types from BRCA2 carriers show wild-type chromosome deletions on 13q12-q13. Cancer Res 1995, 55:4830-4832

4. Sharan SK, Morimatsu M, Albrecht U, Lim DS, Regel E, Dinh C, Sands A, Eichele G, Hasty P, Bradley A: Embryonic lethality and radiation hypersensitivity mediated by Rad51 in mice lacking Brca2. Nature 1997, 386:804-810.

5. Yuan SS, Lee SY, Chen G, Song M, Tomlinson GE, Lee EY: BRCA2 is required for ionizing radiation-induced assembly of Rad51 complex in vivo. Cancer Res 1999, 59:3547-3551.

6. Moynahan ME, Cui TY, Jasin M: Homology-directed dna repair, mitomycinc resistance, and chromosome stability is restored with correction of a Brca1 mutation. Cancer Res 2001, 61:4842-4850.

7. Lomonosov M, Anand S, Sangrithi M, Davies R, Venkitaraman AR: Stabilization of stalled DNA replication forks by the BRCA2 breast cancer susceptibility protein. Genes Dev 2003, 17:3017-3022.

8. Godthelp BC, Wiegant WW, Waisfisz Q, Medhurst AL, Arwert F, Joenje H, Zdzienicka MZ: Inducibility of nuclear Rad51 foci after DNA damage distinguishes all Fanconi anemia complementation groups from D1/BRCA2. Mutat Res 2006, 594:39-48.

9. Lee $S A$, Baker MD: Analysis of DNA repair and recombination responses in mouse cells depleted for Brca2 by SiRNA. DNA Repair (Amst) 2007, 6:809-817.

10. Struewing JP, Hartge $P$, Wacholder S, Baker SM, Berlin M, McAdams M, Timmerman MM, Brody LC, Tucker MA: The risk of cancer associated with specific mutations of BRCA1 and BRCA2 among Ashkenazi Jews. N Engl J Med 1997, 336:1401-1408.

11. Bièche I, Noguès C, Lidereau R: Overexpression of BRCA2 gene in sporadic breast tumours. Oncogene 1999, 18:5232-5238.

12. Venkitaraman AR: Cancer susceptibility and the functions of BRCA1 and BRCA2. Cell 2002, 108:171-182.

13. Egawa C, Miyoshi Y, Taguchi T, Tamaki Y, Noguchi S: High BRCA2 mRNA expression predicts poor prognosis in breast cancer patients. Int $\mathrm{J}$ Cancer 2002, 98:879-882. 
14. Bièche I, Tozlu S, Girault I, Lidereau R: Identification of a three-gene expression signature of poor-prognosis breast carcinoma. Mol Cancer 2004, 3:37.

15. Magwood AC, Mundia MM, Baker MD: High levels of wild-type BRCA2 suppress homologous recombination. J Mol Biol 2012, 421:38-53.

16. Gudmundsdottir K, Lord CJ, Witt E, Tutt AN, Ashworth A: DSS1 is required for RAD51 focus formation and genomic stability in mammalian cells. EMBO Rep 2004, 5:989-993.

17. Wilmes GM, Bergkessel M, Bandyopadhyay S, Shales M, Braberg H, Cagney G, Collins SR, Whitworth GB, Kress TL, Weissman JS, Ideker T, Guthrie C Krogan NJ: A genetic interaction map of RNA-processing factors reveals links between Sem1/Dss1-containing complexes and mRNA export and splicing. Mol Cell 2008, 32:735-746.

18. Ohta K, Kuwahara K, Zhang Z, Makino K, Komohara Y, Nakamura H, Kuratsu J, Sakaguchi N: Decreased expression of germinal center-associated nuclear protein is involved in chromosomal instability in malignant gliomas. Cancer Sci 2009, 100:2069-2076.

19. Nakaya T, Kuwahara K, Ohta K, Kitabatake M, Toda T, Takeda N, Tani T, Kondo E, Sakaguchi N: Critical role of Pcid2 in B cell survival through the regulation of MAD2 expression. J Immunol 2010, 185:5180-5187.

20. Wei SJ, Williams JG, Dang H, Darden TA, Betz BL, Humble MM, Chang FM, Trempus CS, Johnson K, Cannon RE, Tennant RW: Identification of a specific motif of the DSS1 protein required for proteasome interaction and p53 protein degradation. J Mol Biol 2008, 383:693-712.

21. Kim HS, Yom CK, Kim HJ, Lee JW, Sohn JH, Kim JH, Park YL, Ahn SH: Overexpression of $\mathrm{p} 53$ is correlated with poor outcome in premenopausal women with breast cancer treated with tamoxifen after chemotherapy. Breast Cancer Res Treat 2010, 121:777-788.

22. Pulyaeva H, Bueno J, Polette M, Birembaut P, Sato H, Seiki M, Thompson EW: MT1-MMP correlates with MMP-2 activation potential seen after epithelial to mesenchymal transition in human breast carcinoma cells. Clin Exp Metastasis 1997, 15:111-120.

23. Li J, Zou C, Bai Y, Wazer DE, Band V, Gao Q: DSS1 is required for the stability of BRCA2. Oncogene 2006, 25:1186-1194.

24. Yang G, Mercado-Uribe I, Multani AS, Sen S, Shih IM, Wong KK, Gershenson DM, Liu J: RAS promotes tumorigenesis through genomic instability induced by imbalanced expression of Aurora-A and BRCA2 in midbody during cytokinesis. Int J Cancer 2013, 133:275-285.

25. Ma YY, Lin H, Chang FM, Chang TC, Trieu T, Pridgen HI, Zhang Y, Huang J, Patiño-Guzman K, Diab N, Cantu A, Slaga TJ, Wei SJ: Identification of the deleted in split hand/split foot 1 protein as a novel biomarker for human cervical cancer. Carcinogenesis 2013, 34:68-78.

26. Roy R, Chun J, Powell SN: BRCA1 and BRCA2: different roles in a common pathway of genome protection. Nat Rev Cancer 2012, 12:68-78.

27. Xia F, Taghian DG, DeFrank JS, Zeng ZC, Willers H, Iliakis G, Powell SN: Deficiency of human BRCA2 leads to impaired homologous recombination but maintains normal nonhomologous end joining. Proc Natl Acad Sci USA 2001, 98:8644-8649.

28. Chapman JR, Taylor MR, Boulton SJ: Playing the end game: DNA double-strand break repair pathway choice. Mol Cell 2012, 47:497-510.

29. Moynahan ME, Jasin M: Mitotic homologous recombination maintains genomic stability and suppresses tumorigenesis. Nat Rev Mol Cell Biol 2010, 11:196-207.

30. Hanawalt PC, Spivak G: Transcription-coupled DNA repair: two decades of progress and surprises. Nat Rev Mol Cell Biol 2008, 9:958-970.

31. Negrini S, Gorgoulis VG, Halazonetis TD: Genomic instability-an evolving hallmark of cancer. Nat Rev Mol Cell Biol 2010, 11:220-228.

32. Jani D, Lutz S, Hurt E, Laskey RA, Stewart M, Wickramasinghe VO: Functional and structural characterization of the mammalian TREX-2 complex that links transcription with nuclear messenger RNA export. Nucleic Acids Res 2012, 40:4562-4573.

33. Wickramasinghe VO, McMurtrie PI, Mills AD, Takei Y, Penrhyn-Lowe S, Amagase $Y$, Main S, Marr J, Stewart M, Laskey RA: mRNA export from mammalian cell nuclei is dependent on GANP. Curr Biol 2010, 20:25-31.

34. Sinha BK, Haim N, Dusre L, Kerrigan D, Pommier Y: DNA strand breaks produced by etoposide (VP-16,213) in sensitive and resistant human breast tumor cells: implications for the mechanism of action. Cancer Res $1988,48: 5096-5100$

35. Liu YY, Yu JY, Yin D, Patwardhan GA, Gupta V, Hirabayashi Y, Holleran WM, Giuliano AE, Jazwinski SM, Gouaze-Andersson V, Consoli DP, Cabot MC: A role for ceramide in driving cancer cell resistance to doxorubicin. FASEB $J$ 2008, 22:2541-2551.

36. Pegg AE: Mammalian O6-alkylguanine-DNA alkyltransferase: regulation and importance in response to alkylating carcinogenic and therapeutic agents. Cancer Res 1990, 50:6119-6129.

37. Lage H, Dietel M: Involvement of the DNA mismatch repair system in antineoplastic drug resistance. J Cancer Res Clin Oncol 1999, 125:156-165.

38. Lage $H$, Dietel M: Effect of the breast-cancer resistance protein on atypical multidrug resistance. Lancet Oncol 2000, 1:169-175.

39. Egawa C, Miyoshi Y, Takamura Y, Taguchi T, Tamaki Y, Noguchi S: Decreased expression of BRCA2 mRNA predicts favorable response to docetaxel in breast cancer. Int J Cancer 2001, 95:255-259.

40. Rytelewski M, Ferguson PJ, Maleki Vareki S, Figueredo R, Vincent M, Koropatnick J: Inhibition of BRCA2 and thymidylate synthase creates multidrug sensitive tumor cells via the induction of combined "complementary lethality". Mol Ther Nucleic Acids 2013, 2:e78.

doi:10.1186/1471-2407-13-562

Cite this article as: Rezano et al:: Breast cancers with high DSS1 expression that potentially maintains BRCA2 stability have poor prognosis in the relapse-free survival. BMC Cancer 2013 13:562.

\section{Submit your next manuscript to BioMed Central and take full advantage of:}

- Convenient online submission

- Thorough peer review

- No space constraints or color figure charges

- Immediate publication on acceptance

- Inclusion in PubMed, CAS, Scopus and Google Scholar

- Research which is freely available for redistribution 